\title{
A Synthesis of Optimization Approaches for LCA-Integrated Industrial Process Modeling: Application to Potable Water Production Plants
}

\author{
Florin Capitanescu, Antonino Marvuglia and Enrico Benetto
}

\begin{abstract}
This paper synthesizes the authors' experience in the area of integrated approaches coupling multi-objective optimization (MOO), industrial process modeling and simulation, and life cycle assessment (LCA), with particular application to the sector of drinking water production. An industrial process is intended as any process using a certain technology to produce a product or deliver a service. The paper discusses comparatively the suitability for the optimization of a real-world drinking water production plant (DWPP) of four optimization approaches, namely: (1) off-the-shelf global search metaheuristic algorithms, (2) hybrid optimizers combining global search and local search, (3) surrogate model based optimizers, and (4) local search.
\end{abstract}

\section{Introduction}

The combination between various optimization methods and life cycle assessment (LCA) has been initiated two decades ago [1], with the aim to empower decision makers with Pareto trade-off cost-effective solutions to decrease environmental impacts of processes. Many approaches have been proposed since then (e.g. [2-4]) in this research area; the reader is referred to [5] for a relatively comprehensive survey. Rooted in the same research field, but focusing on the computationally expensive optimization problem of eco-design of drinking water production plants (DWPPs), the 3-year project "Optimization based integrated process modellingLCA: application to potable water production" (OASIS) has further explored the best paths for the threefold coupling (process modeling, LCA and optimization) along four major optimization research streams namely: (1) off-the-shelf global search metaheuristic optimization algorithms [6], (2) hybrid optimizers combining global search and local search [7], (3) surrogate model based optimizers [8, 9], and (4) local search [10].

\footnotetext{
F. Capitanescu $(\square) \cdot$ A. Marvuglia $\cdot$ E. Benetto

Luxembourg Institute of Science and Technology (LIST), Belvaux, Luxembourg

e-mail: florin.capitanescu@ list.lu

(C) The Author(s) 2018

E. Benetto et al. (eds.), Designing Sustainable Technologies,

Products and Policies, https://doi.org/10.1007/978-3-319-66981-6_3
} 
The outline of the remaining of the paper is as follows. Section 2 presents the statement of the problem and the tool components. Section 3 briefly describes the main optimization algorithms employed in the four research streams. Section 4 provides optimization results with these algorithms for a realistic model of a real-world DWPP. Section 5 concludes and provides directions for future work.

\section{Statement of the Optimization-Process Modelling-LCA Problem}

\subsection{Problem Formulation}

The multi-objective optimization (MOO) problem corresponding to a DWPP can be compactly expressed, assuming a single relevant aggregated operating scenario, as follows:

$$
\begin{array}{cc}
\min _{\mathbf{x}} & \left\{f_{1}(\mathbf{x}), f_{2}(\mathbf{x})\right\} \\
\text { subject to: } & \mathbf{g}(\mathbf{x})=\mathbf{0} \\
& \mathbf{h}(\mathbf{x}) \geq \underline{\mathbf{h}} \\
& \underline{\mathbf{x}} \leq \mathbf{x} \leq \overline{\mathbf{x}}
\end{array}
$$

where: $\mathbf{x}$ denotes the vector of decision variables (e.g. design and operation parameters of the DWPP unit processes), $f_{1}$ models the operation cost of the DWPP (comprising especially raw materials, chemicals, and electricity), $f_{2}$ models the environmental impacts of the DWPP (calculated using ReCiPe method applied at midpoint level [11]). The equality constraints $\mathbf{g}(\mathbf{x})=\mathbf{0}$ model the input-output mass flow for each unit process in the entire chain. The inequality constraints $\mathbf{h}(\mathbf{x}) \geq \underline{\mathbf{h}}$ enforce the outlet water quality [6]. The latter is represented only by seven relevant aggregated parameters (e.g. total coliforms, total trihalomethanes, total organic carbon, Escherichia coli, faecal streptococci, turbidity, and conductivity). Finally, the inequality constraints $\underline{\mathbf{x}} \leq \mathbf{x} \leq \overline{\mathbf{x}}$ model the physical bounds of the decision variables.

Note that, because there is no qualitative or quantitative benefit to express analytically the hundreds of complex chemical reactions involved in the optimization problem (1), these are assessed (by the specialized software PHREEQC ${ }^{\circledR}[12]$ ) by running the DWPP simulator, called EVALEAU [13], for specific values of the decision variables. 


\subsection{EVALEAU: The DWPP Simulator}

EVALEAU is a simulator of DWPPs (see Fig. 1) which integrates detailed modelling of DWPPs unit operations with state of the art LCA tools [13]. It consists in a library of unit process (UPs) models for water treatment, allowing life cycle inventory calculation (LCI) as a function of process parameters. The tool is embedded in the LCA software Umberto ${ }^{\circledR}$ and relies on the Ecoinvent database. It also includes a sensitivity analysis toolbox based on the Morris method for the identification of the process parameters mainly affecting the life cycle impact assessment (LCIA) results. More details about it can be found in the literature [13-15].

\subsection{The Proposed Integrated Simulation-Optimization Approach}

The approach proposed in the OASIS project for solving the simulationoptimization problem (1) consists in decomposing the problem into two modules (the EVALEAU simulator and a multi-objective optimizer) which interact in a loop, as shown in Fig. 2.

Because the simulator is intrinsically computationally expensive (it takes in average around two minutes per simulation) and can be seen by the optimizer as a black-box, suitable optimizers have been sought as described in the next section.

\section{Optimization Approaches}

While the benefits of using optimization techniques for DWPP eco-design (e.g. reduction in both operating cost and environmental impacts) have been quantified in our previous works (e.g. [6-10, 16]), this section summarizes chronologically the main four lines of research pursued in the OASIS project, whose performances will be compared in the next section.



Fig. 1 Modular model of a real-world DWPP (I, O and T stand for input, output, and unit process, respectively) 




Fig. 2 Architecture of the integrated tool coupling the DWPP EVALEAU simulator with an optimizer

\subsection{Off-the-Shelf Global Search Metaheuristic Algorithms}

Global search generic metaheuristic algorithms have been naturally the first optimizers tested. The detailed results obtained with six algorithms of this class have been reported in [6]. Two algorithms, namely the Strength Pareto Evolutionary Algorithm (SPEA2) [17] and the Non-dominated Sorting Genetic Algorithm (NSGA-II) [18], have proven consistently best performances in terms of convergence speed to the Pareto front. However, these algorithms involve heavy computations, due to their inherent slow convergence near to the optimum and genericity (i.e. they make no attempt to take advantage of the problem structure). This fact motivates further research among the three following lines.

\subsection{Hybrid Algorithms Combining Global and Local Search}

Hybrid algorithms combine global search (or exploration) and local search (or exploitation) so as to take advantage of their assets while offsetting their drawbacks.

Two coupling schemes between global search and local search have been used:

(1) sequential approach (explored to some extent in [10]): global search identifies first the most promising regions of the design space and then its final solutions are transferred to the local search method (described in Sect. 3.4) for further local refinement;

(2) integrated approach (explored in [7]): the local search module is embedded in the global search algorithm; the local search is called at a certain pace to improve locally the best current candidate solutions. Specifically, [7] has 
proposed such a hybrid approach in which a memory-based adaptive partitioning algorithm was embedded into an archive-based multi-objective evolutionary algorithm, developed relying on the structure of NSGA II [18].

\subsection{Surrogate Model-Based Optimizers}

This research line consists in building tailored computationally cheap surrogate models of the optimization problem, aiming to accelerate the convergence and reduce the computational burden. Two approaches have been developed $[8,9]$ in which the surrogate model of the optimization problem is based on:

(1) mixed-integer linear programming (MILP) $[8,16]$. This surrogate model relies on piecewise linear approximations, via brute-force sensitivity computation, of the objective functions and inequality constraints. Additionally, the use of constraint programming [19] for solving the MILP problem at the core of the surrogate model has been explored in [9].

(2) nonlinear programming (NLP). This surrogate model relies on curve fitting of objectives and inequality constraints via either quadratic polynomial functions or higher order polynomial functions (e.g. cubic) [9].

In both proposed methodologies which include such surrogate models, the approximation of the Pareto front is generated upon applying the well-known $\varepsilon$-constraint method [20] to the MOO surrogate problems.

\subsection{Local Search}

The last optimization research strand investigated in this project concerns the local search [10]. The latter is useful not only in the context of hybrid algorithms (see Sect. 3.2) but also in many real-world computationally expensive simulation-based applications, where the aim is to improve a given system state locally with limited computational budget. To this end, a new neighborhood-based iterative local search method has been proposed [10]. This method aims at steering the search along any desired direction in the objectives space and resorting to first derivatives approximation and linear programming optimization. 


\section{Numerical Results}

\subsection{Short Description of the DWPP}

The optimization approaches developed in the frame of the OASIS project have been applied to a realistic model of an existing DWPP from France [13]. The DWPP treatment chain of the inlet river water contains the main unit process (see Fig. 1): pumping, a first ozonation, coagulation/flocculation, settling, biolite filtration, a second ozonation, granular activated carbon filtration, and bleach disinfection. A set of six relevant decision variables is considered, namely (see Fig. 1): the ozone transfer efficiency and the pure oxygen fraction in feed gas in units T4 and $\mathrm{T} 17$, the coagulant dose in unit $\mathrm{T} 7$, and the granular activated carbon regeneration frequency in unit $\mathrm{T} 20$.

\subsection{Results Using off-the-Shelf Global Search Algorithms}

Figures 3 shows three snapshots retrieved during the convergence to the Pareto front obtained with SPEA2 and NSGA-II algorithms. For all the algorithms the Pareto front corresponds to roughly 50 generations of simulation.

By comparing the two sides of Fig. 3 one can note that, while after 10 generations NSGA-II front approximation is substantially better (in terms of non-dominance and spread of solutions) than the one of SPEA2, as generations evolve, SPEA2 slightly outperforms NSGA-II.
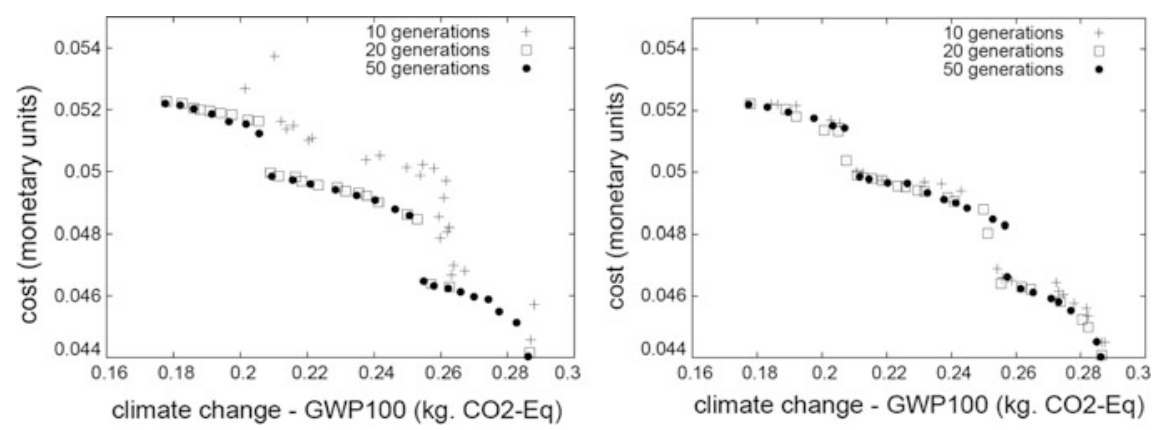

Fig. 3 Progress toward the Pareto front obtained with SPEA2 (left) and NSGA II (right) optimizers [6] 


\subsection{Results Using Hybrid Algorithms}

Figures 4 and 5 present results obtained with two hybrid algorithms: a sequential two step algorithm (Fig. 4) and an integrated algorithm (Fig. 5).

Figure 4 shows that, although the local search is launched (starting from best solutions provided by the global search algorithm SPEA2 after 120 evaluations) relatively far from the Pareto front, despite the limited budget, it behaves well (i.e. a few solutions converge already on the front and their spread is good) [10].

Figure 5 shows the results obtained with an integrated hybrid algorithm called Archive-based Multi-Objective Evolutionary Algorithm with Memory-based Adaptive Partitioning of search space (AMOEA-MAP) [7]. Despite the limited computational budget allowed (200 evaluations) this algorithm exhibits excellent performances compared to NSGA-II. It also outperforms the sequential algorithm.

Fig. 4 Solution path of the local search method applied after 120 evaluations of global search algorithm SPEA2 [8]
Fig. 5 Approximation of the Pareto front as a function of the number of simulator evaluations: hybrid algorithm (AMOEA-MAP) versus NSGAII optimizer [7]
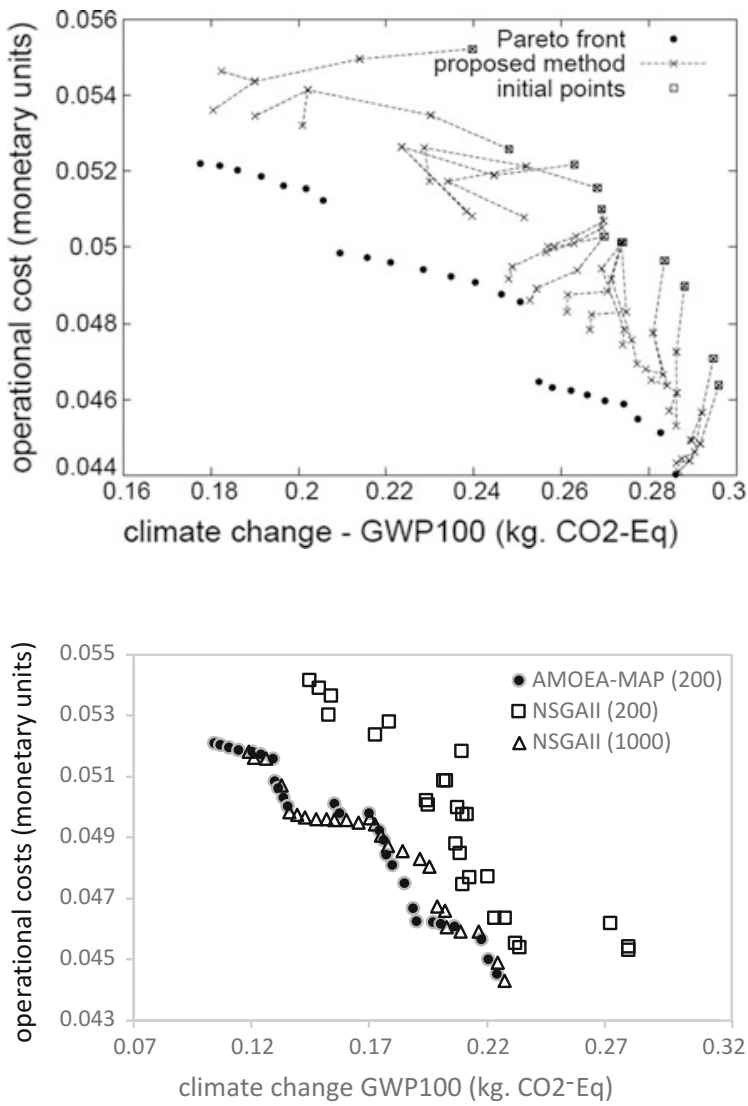


\subsection{Results Using Surrogate Model-Based Optimizers}

Figure 6 plots the front approximations obtained with the MILP-based surrogate model using 10 function linearization intervals [8]. One can observe that, for the same number of evaluations, the MILP-based algorithm outperforms SPEA2.

Due to space limitation, the results obtained with NLP-based surrogate models [8, 9] and constraint programming [16], both performing less well than MILP model, are not shown. For detailed results the reader is referred to $[8,9,16]$.

\subsection{Results Using Local Search}

Figure 7 displays the solution path obtained with the LP-based local search method [10], starting from an initial operating point where each decision variable is set to the half value of its physical range. One can first observe that the method has a good ability to steer the search along the desired direction in the objectives space. Furthermore, the method produces generally a high quality approximation of the Pareto front (in terms of accuracy and distribution of solutions), especially in the upper concave part of the front, except of one search trajectory in the middle which gets stuck.

Fig. 6 Approximation of the Pareto front for MILP-based surrogate algorithm [8]

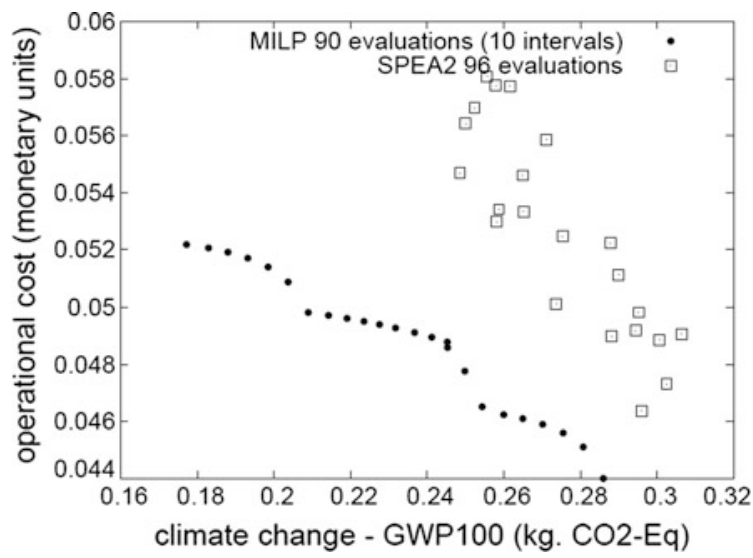


Fig. 7 Solution path and approximated Pareto front via the LP-based local search method [10]

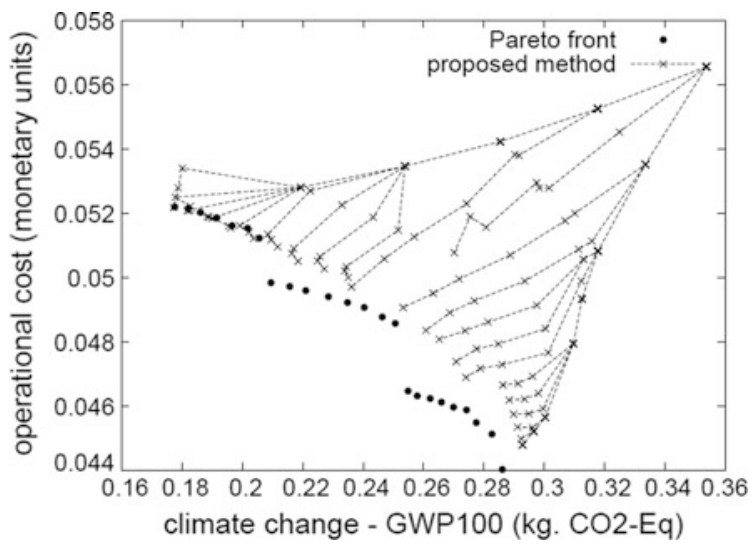

\section{Conclusions and Outlook}

This paper has synthesized the main findings of our experiments with four classes of optimization approaches for the MOO of the DWPP in the frame of the project OASIS. The main conclusions drawn can be summarized as follows.

(1) Hybrid algorithms, and particularly the integrated hybrid algorithm AMOEAMAP, and MILP-based surrogate model have shown the best performances, compared to other competing alternatives.

(2) The off-the-shelf generic global search metaheuristic algorithms are substantially less efficient than the best classes of algorithms. However, given especially their application straightforwardness, these alternatives cannot be discarded. Among the tested algorithms, NSGA-II has shown the best performances overall, being closely followed by SPEA2. The experiments with four other popular metaheuristic algorithms have indicated that, in the myriad of existing meta-heuristic algorithms with various pros and cons, the best algorithm for a given problem should be chosen carefully.

(3) Constraint (integer) programming is less suitable than MILP classical algorithms in our context of loosely constrained small size surrogate optimization problem, where feasibility is not a major concern.

(4) The proposed LP-based local search method has shown good performances and keeps intact its promises for other mildly nonlinear computationally expensive optimization problems.

Although the explored optimization algorithms have been applied to the bi-objective (e.g. cost versus LCA-based environmental impact) optimization of DWPP at planning stage, they remain generic to other application fields dealing with (computationally expensive) MOO problems. Furthermore, our results with these algorithms could serve to evaluate their suitability for different problems. In our experiments we have noticed that the water quality constraints are not severely 
constraining the feasible region of the problem; this is however case-dependent and the impact of these constraints may be more pronounced for other DWPPs.

As a side complementary experiment of the project, a detailed discussion regarding the pros and cons of expressing environmental impacts in MOO utilizing the midpoint categories versus endpoint score has been conducted in [21].

Finally, the algorithms developed and the experience gained in this project, could be applied (with due adaptations) to other case studies. In particular, a very appealing and timely research area would be the optimization of supply chains, under resiliency constraints and risk-based decision making.

Acknowledgements The authors acknowledge the funding from Luxembourg National Research Fund (FNR) in the framework of the OASIS project (CR13/SR/5871061).

\section{References}

1. A. Azapagic, R. Clift, Life cycle assessment and multiobjective optimisation, Journal of Cleaner Production, Vol. 7, 1999, pp. 135-143.

2. I.E. Grossman, G Guillén-Gosálbez, Scope for the Application of Mathematical Programming Techniques in the Synthesis and Planning of Sustainable Processes, Computers \& Chemical Engineering, Vol. 34, 2010, pp. 1365-1376.

3. G. Guillén-Gosálbez, J.A. Caballero, L. Jiménez, Application of Life Cycle Assessment to the Structural Optimization of Process Flowsheets, Industrial \& Engineering Chemistry Research, Vol. 47, 2008, pp. 777-789.

4. F. You, L. Tao, D.J. Graziano, S.W. Snyder, Optimal design of sustainable cellulosic biofuel supply chains: Multiobjective optimization coupled with life cycle assessment and inputoutput analysis, AIChE Journal, Vol. 58, 2012, pp. 1157-1180.

5. C. Pieragostini, M.C. Mussati, $P$ Aguirre, On process optimization considering LCA methodology, Journal of Environmental Management, Vol. 96, 2012, pp. 43-54.

6. F. Capitanescu, S. Rege, A. Marvuglia, E. Benetto, A. Ahmadi, T. Navarrete-Gutierrez, L. Barna, Cost versus life cycle assessment-based optimization of drinking water production plants, Journal of Environmental Management, Vol. 177, 2016, pp. 278-287.

7. A. Ahmadi, L. Barna, F. Capitanescu, A. Marvuglia, E. Benetto, An archive-based multi-objective evolutionary algorithm with adaptive search space partitioning to deal with expensive optimisation problems: application to process eco-design, Computers \& Chemical Engineering, Vol. 87, 2016, pp. 95-110.

8. F. Capitanescu, A. Ahmadi, E. Benetto, A. Marvuglia, L. Barna, Some efficient approaches for multi-objective constrained optimization of computationally expensive black-box model problems, Computers \& Chemical Engineering, Vol. 82, No. 2, 2015, pp. 228-239.

9. F. Capitanescu, A. Marvuglia, E. Benetto, A. Ahmadi, L. Barna, Assessing the uses of NLP-based surrogate models for solving expensive multi-objective optimization problems: application to potable water chains, Enviroinfo Conference, Copenhagen (Denmark), 2015.

10. F. Capitanescu, A. Marvuglia, E. Benetto, A. Ahmadi, L. Barna, Linear programming-based directed local search for expensive multi-objective optimization problems: application to drinking water production plants, European Journal of Operational Research, Vol. 262, 2017, pp. 322-334.

11. M.J. Goedkoop, R. Heijungs, M. Huijbregts, A. De Schryver, J. Struijs, R. Van Zelm, ReCiPe 2008-A life cycle impact assessment method which comprises harmonised category indicators at the midpoint and the endpoint level, First edition Report I: Characterisation, 6 January 2009. 
12. D.L. Parkhurst, C.A.J. Appelo, User's guide to PHREEQC (version 2)-A computer program for speciation, batch-reaction, one-dimensional transport, and inverse geochemical calculations. U.S.G.S., 1999.

13. Y. Méry, L. Tiruta-Barna, E. Benetto, I. Baudin, An integrated "process modelling-life cycle assessment" tool for the assessment and design of water treatment processes, International Journal of Life Cycle Assessment, Vol. 18, 2013, pp. 1062-1070.

14. Y. Méry, L. Tiruta-Barna, I. Baudin, E. Benetto, E. Igos, Formalization of a technical procedure for process ecodesign dedicated to drinking water treatment plants, Journal of Cleaner Production, Vol. 68, 2014, pp. 16-24.

15. Y. Méry, Development of an integrated tool for Process Modelling and Life Cycle Assessment-Ecodesign of process plants and application to drinking water treatment. Chemical and Process Engineering. INSA de Toulouse, 2012. English.

16. F. Capitanescu, A. Marvuglia, E. Benetto, A. Ahmadi, L. Barna, Constraint Programming versus MIP for LCA-based Multi-Objective Optimization of Sustainable Potable Water Production Plant, iEMSs Conference, Toulouse (France), 2016.

17. E. Zitzler, M. Laumanns, L. Thiele, SPEA2: Improving the Strength Pareto Evolutionary Algorithm for Multiobjective Optimization, In: Evolutionary Methods for Design, Optimisation and Control with Application to Industrial Problems (Eds. Giannakoglou K.C. et al.), Proceedings of the EUROGEN2001 Conference, Athens, Greece, 2002, pp. 95-100.

18. K. Deb, S. Agrawal, A. Pratap, T. Meyarivan, A Fast Elitist Non-dominated Sorting Genetic Algorithm for Multi-objective Optimization: NSGA-II, PPSN VI, LNCS, 1917, 2000, pp. 849-858.

19. T. Achterberg, SCIP: solving constraint integer programs, Mathematical Programming Computation, 2009, pp. 1-41.

20. G. Mavrotas, Effective implementation of the $\varepsilon$-constraint method in Multi-Objective Mathematical Programming problems. Applied Mathematics and Computation, 2009; Vol. 2013, pp. 455-65.

21. F. Capitanescu, E. Igos, A. Marvuglia, E. Benetto, Midpoint vs single score in multi-criteria optimization under life cycle assessment constraints: the case of potable water treatment chains, Enviroinfo Conference, Copenhagen (Denmark), 2015.

Open Access This chapter is licensed under the terms of the Creative Commons Attribution 4.0 International License (http://creativecommons.org/licenses/by/4.0/), which permits use, sharing, adaptation, distribution and reproduction in any medium or format, as long as you give appropriate credit to the original author(s) and the source, provide a link to the Creative Commons license and indicate if changes were made.

The images or other third party material in this chapter are included in the chapter's Creative Commons license, unless indicated otherwise in a credit line to the material. If material is not included in the chapter's Creative Commons license and your intended use is not permitted by statutory regulation or exceeds the permitted use, you will need to obtain permission directly from the copyright holder.

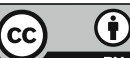

